Advancements in Instrumentation

\title{
Microscopic Measurement of Circular Dichroism Spectra
}

\author{
Aira Matsugaki, Hideaki Takechi, Hideaki Monjushiro, and Hitoshi Watarai ${ }^{\dagger}$ \\ Department of Chemistry, Graduate School of Science, Osaka University, Toyonaka, Osaka 560-0043, Japan
}

\begin{abstract}
A microscope device to measure the circular dichroism (CD) spectra of a specified microscopic region of chiral samples was constructed by combining of a couple of objective lenses and a CCD camera, which was installed in a sample chamber of a commercially available CD spectropolarimeter. By using this apparatus, high quality micro-CD spectra in the $60 \times 60 \mu \mathrm{m}$ region of samples could be measured. Micro-CD spectra of thin film of chiral DNA samples on glass and a natural kidney bean leaf were measured, and the potential of the micro-CD apparatus was successfully demonstrated.
\end{abstract}

(Received October 24, 2007; Accepted December 6, 2007; Published February 10, 2008)

\section{Introduction}

The circular dichroism (CD) measurement is now a crucial technique for characterizing and quantifying natural and synthetic chiral systems. Optical chirality is a fundamental property, which provides valuable information on the structure of molecules and the conformation of their assemblies. Therefore, it is widely used in various fields, including organic chemistry, coordination chemistry, and biochemistry..$^{1,2}$

Usually, isotropic and homogeneous solution samples are employed for measuring the CD spectra; however, in some cases CD spectra of samples with unknown inhomogeneity should be measured. For example, we have observed that some chiral aggregates grown at liquid/liquid interfaces show chiral activity, ${ }^{3-8}$ but the chirality of the individual micro-aggregate can not measured by a conventional CD spectropolarimeter. Also, almost all biological samples have inhomogeneity, because they consist of various tissues. It is therefore desired to measure the CD spectra of a micro-size region in the sample of interest.

The idea of a CD microscope for anisotropic samples is not new. In 1982, Maestre and Katz adapted a Cary spectropolarimeter to a microscope for single-point measurements of the CD spectra of chromatin. ${ }^{9}$ Recently, some researchers developed unique microscopic CD measuring methods, including a circular dichroism imaging microscopy, ${ }^{10}$ a circular-dichroism thermal lens microscope ${ }^{11}$ and a fluorescence-detected circular dichroism microscopy. ${ }^{12}$ However, it is still required to measure the reliable CD spectrum of the specified micro-region of films and thin solid samples.

In the present study, we constructed a home-built microscopic device to measure a microscopic region of thin transparent samples, which can be installed in a sample chamber of a commercially available high-performance $\mathrm{CD}$ spectropolarimeter. In the case of an isotropic sample, the observed CD spectrum of a minute domain of the sample was in good agreement with that of the macro-region. Even for inhomogeneous samples, where the macro-CD measurement could not detect any net CD signal, we could observe the optical chirality for a microscopic region of the samples specified by the CCD image.

$\dagger$ To whom correspondence should be addressed.

E-mail:watarai@chem.sci.osaka-u.ac.jp

\section{Experimental}

\section{Materials}

As samples to check the feasibility of the device, we used an oligonuclerotide of $10 \mathrm{mer}$, $\left[\mathrm{d}(\mathrm{AT})_{5}\right]_{2}$, which was prepared from a single-stranded oligomer (Invitrogen Japan, Tokyo) by annealing in a buffer solution including $10 \mathrm{mM}$ Tris- $\mathrm{HCl}, 1 \mathrm{M} \mathrm{NaCl}$ and $10 \mathrm{mM}$ EDTA after heating at $65^{\circ} \mathrm{C}$ for $10 \mathrm{~min}$. The solution of $5 \mu \mathrm{M}$ double-stranded DNA oligomer, $\left[\mathrm{d}(\mathrm{AT})_{5}\right]_{2}$, was stained by a cationic 5,10,15,20-tetrakis $(N$-methylpyridinium-4-yl)$21 H, 23 H$-porphine (TMPyP), whose concentration was varied in the range of $R=0.25-2.0$, where $R$ is defined as the concentration ratio of $[\mathrm{TMPyP}] /[\mathrm{DNA}]$. A film sample of the TMPyP-DNA complex was prepared as follows. Aliquots of $200 \mu \mathrm{l}$ of the TMPyP-stained DNA solution were dropped on a cover glass (MATSUNAMI $18 \times 18 \mathrm{~mm}$ ), which was pretreated by $3 \mathrm{mg} / \mathrm{ml}$ cationic poly(allylamine hydrochloride) (PAH) (Aldrich, $M_{\mathrm{W}} 30000$ ) aqueous solution to improve the affinity to the negatively charged DNA molecules. The solution was spread for about $1 \mathrm{~cm}^{2}$ area, and then dried in a clean bench.

Another sample was a fresh leaf of kidney bean, Phaseolus vulgaris. The leaf was cut in a squared peace of about $10 \times 10$ $\mathrm{mm}$ and sandwiched by a couple of cover glasses.

\section{Microscopic circular dichroism spectral measurements}

Figure 1 shows a schematic illustration of our experimental apparatus, where a beam splitter (Edmund Optics, Japan), two long working distance objective lenses $(\times 20)$ (Mitutoyo, Japan), a mirror (SIGMA KOKI, Japan), a back light (Mega Light 100, HOYA-SCHOTT), a XYZ and rotation stage (SIGMA KOKI, Japan), a tube lens (Mitutoyo, Japan), and a CCD camera (USB 2.0 Camera, Fortissimo, Japan) were arranged on an optical base board of $300 \times 300 \mathrm{~mm}$. The base board was installed in a sample chamber of a CD spectropolarimeter (JASCO J-820) to measure the micro-CD spectra and to obtain an image of the samples in order to specify the observed region. For measuring the micro-CD spectra, the set up shown in Fig. 1(a) was employed. The circularly polarized beam at a frequency of 100 $\mathrm{Hz}$ from a light source was focused on the sample by an objective lens (I). The penetrated beam was collimated by the objective lens (II), and then detected by a photomultiplier with a frequency-control technique to obtain $\mathrm{CD}$ and absorption 
(a)

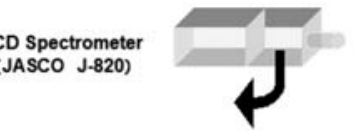

Back Light (off)
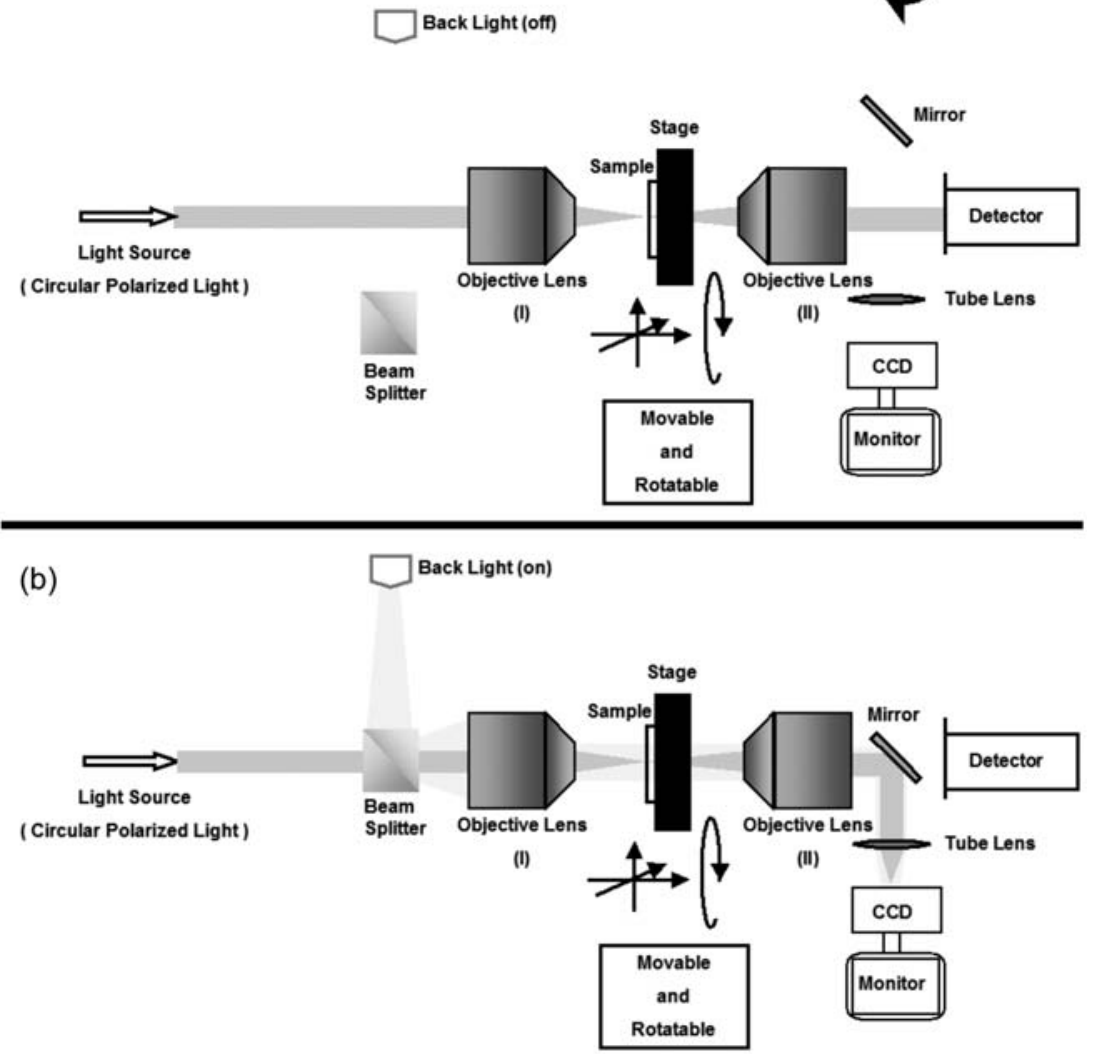

Fig. 1 Schematic illustration of the micro-circular dichroism spectropolarimeter. (a) Set up for a micro-CD measurement and (b) set up for observing sample images by CCD.

spectra of microscopic region of the sample. The sample was vertically fixed on the position-orientation controllable stage to adjust the sample position. In order to specify the observed region and the beam size on the sample, the CCD image of the sample plane was obtained by using the set up shown in Fig. 1(b). In this case, the beam splitter and the mirror were introduced into the beam path and the CCD image of the sample was observed through the objective lens (II). Under the condition of the present study, the irradiation area by the incident beam at the sample position was about $9 \times 9 \mathrm{~mm}$, but it was reduced to $60 \times 60 \mu \mathrm{m}$ after focusing with the objective lens $(\times 20)$. The size of the irradiation area was determined by the size of the exit slit of the monochromator equipped in the spectropolarimeter as well as the magnification power of the objective lens. Therefore, the irradiation area became small by reducing the size of the exit slit, but this caused the decrease in the intensity of the irradiation beam. In order to obtain a highquality micro-CD spectrum, we used a slit size of $500 \times 500 \mu \mathrm{m}$ for the measurement. We could measure the macro-CD spectra of the sample in the area of $9 \times 9 \mathrm{~mm}$ in the absence of the objective lenses as well as micro-CD spectra in the area of $60 \times$ $60 \mu \mathrm{m}$ with the objective lenses. All of the presented CD spectra were measured under the conditions of $0.5 \mathrm{~nm}$ intervals, a $50 \mathrm{~nm} / \mathrm{min}$ scan speed, and a $0.25-\mathrm{s}$ response time.

\section{Results and Discussion}

Figure 2 shows the macro-CD and absorption spectra of the 5 $\mu \mathrm{M}$ double-stranded DNA oligomer solutions with different concentrations of TMPyP. It was observed that the CD signal was positive under the low TMPyP concentration conditions of $R=0.25-0.75$, and the CD spectrum became bisignate, overlapped by positive signals when TMPyP concentration was increased $(R=1.0-2.0)$. The three major binding modes for the porphyrin-DNA complexes have been generally accepted, namely, intercalation, outside bonding and groove binding; these three binding modes were distinguished by the shape of the CD spectrum. ${ }^{13-17}$ According to these studies, it was suggested that the interaction mode of TMPyP with DNA was the groove binding under the conditions of $R=0.25-0.75$, since the $C D$ signal was positive. On the other hand, when $R=1.0-2.0$, the CD spectra showed the bisignate overlapped by positive signals, which suggested the existents of both the groove binding and the outside binding interaction modes.

The dry-up film sample of TMPyP without DNA on the cover glass showed a maximum absorption at $425 \mathrm{~nm}$ in the absorption spectrum, but no chiral signal was obserevd in both the macroand micro-CD spectra. However, the dried film sample of the DNA-TMPyP complex on glass gave CD signals in the micro$\mathrm{CD}$ spectrum. The intensities of the absorption and the CD signals depended on the concentration of TMPyP, and also on the observing position on the sample film. Figure 3 shows examples of the micro-CD and the absorption spectra of the DNA-TMPyP complex $(R=2.0)$ film obtained at different sample positions. At position (a) shown in Fig. 3, the DNATMPyP film gave a definite bisignate $\mathrm{CD}$ signal due to the exciton coupling chirality of outside binding TMPyP to DNA, 
accompanied by the absorption maximum at $433 \mathrm{~nm}$ (Fig. 3(c)) While at the position (b) shown in Fig. 3, a simple positive CD signal was observed (Fig. 3(d)). This observation indicates that

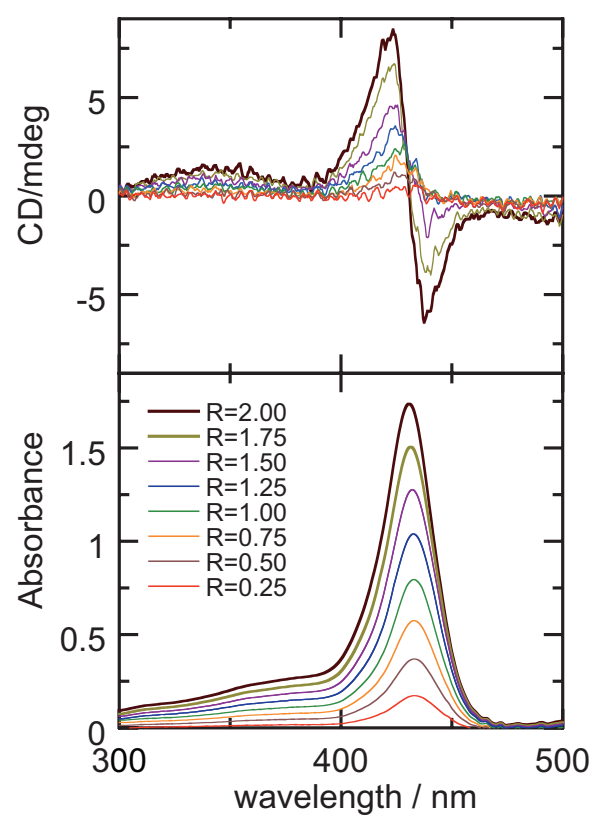

Fig. $2 \mathrm{CD}$ and absorption spectra of DNA-TMPyP in aqueous solution at different concentration ratios. $R$ is the concentration ratio of [TMPyP]/[DNA]. the interaction mode of TMTyP with DNA in the region is mainly the groove binding mode. These results indicate that the chirality of the prepared DNA-TMPyP film was inhomogeneous, and the constructed micro-CD apparatus could characterize the local chirality of the sample. The CD signal was very weak, when the whole area of $9 \times 9 \mathrm{~mm}$ of the sample film was measured. This is due to an inhomogeneity in the thickness of the sample film as well as an averaging effect of the various local chiralities.

In Fig. 4, the measurement of the second sample is demonstrated: the micro-CD and absorption spectra of the leaf blade and the leaf vein of kidney bean, Phaseolus vulgaris. The micro-CD and absorption spectra of the blade part of the leaf (Figs. 4(a), (c)) have two peaks at about $660 \mathrm{~nm}$ and around 400 $-500 \mathrm{~nm}$. It is well known that the absorption spectra of chlorophylls have two peaks at about 660 and $450 \mathrm{~nm} .^{18,19}$ It is therefore thought that the observed spectra of the leaf blade mainly come from chlorophylls, which undergo an interaction with $\alpha$ helix. However, the $\mathrm{CD}$ and absorption spectra of the leaf vein (Figs. 4(b), (d)) have no peaks that corresponds to chlorophylls, because the leaf vein has no chlorophyll in the part of a vascular bundle, running in a leaf blade. Photosynthesis is not carried out in the leaf vein. We also observed the macro$\mathrm{CD}$ and absorption spectra of a $9 \times 9 \mathrm{~mm}$ area of a leaf of kidney bean after removing the microscopic device. The macro-CD and absorption spectra were similar to the micro-CD and absorption spectra of the leaf blade. This is because the relative area of the leaf blade part in the whole leaf was much higher than that of the leaf vain. These results were therefore appropriate. Also, the validity of the microspectropolarimeter could be checked by comparing these spectra. (a)

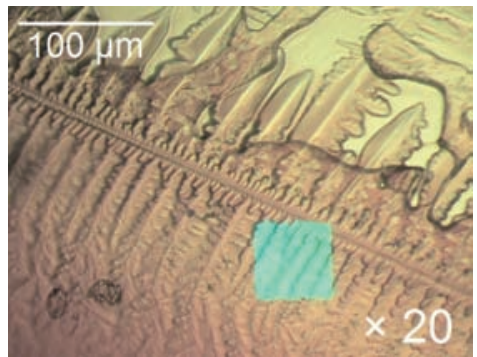

(c)

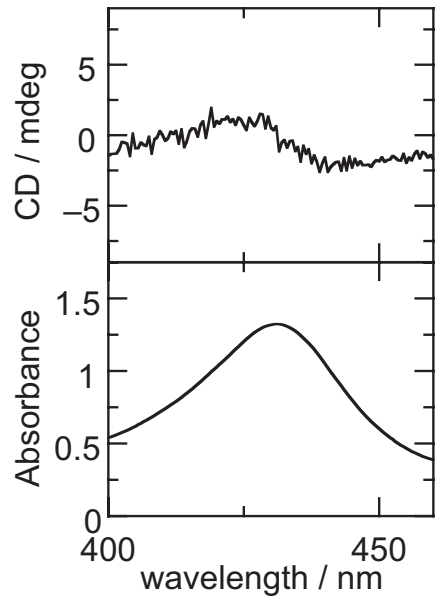

(b)

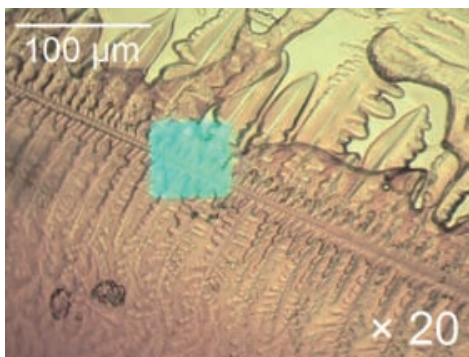

(d)

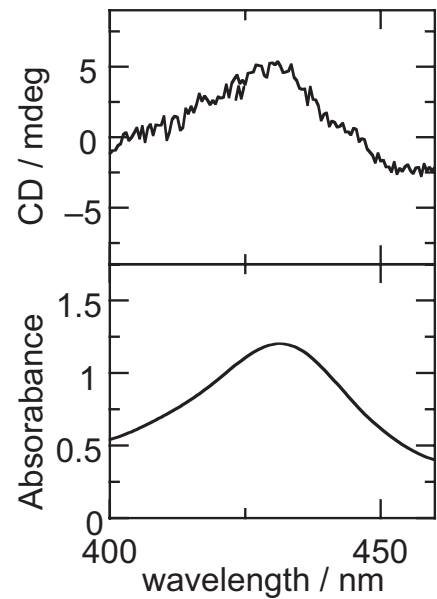

Fig. 3 CCD images, micro-CD and absorption spectra of dried film sample of the DNA-TMPyP complex on a glass substrate. (a) and (b) Images of DNA-TMPyP film and the micro-CD measurement regions (the green-colored areas in the images), (c) micro-CD and absorption spectra of the region indicated in (a), (d) micro-CD and absorption spectra of the region indicated in (b). 
(a)

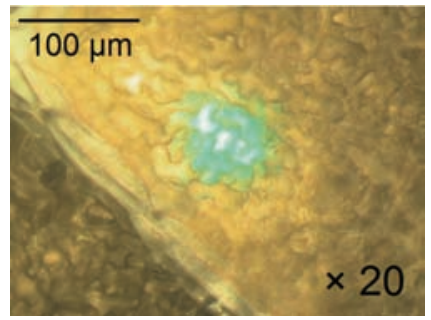

(c)

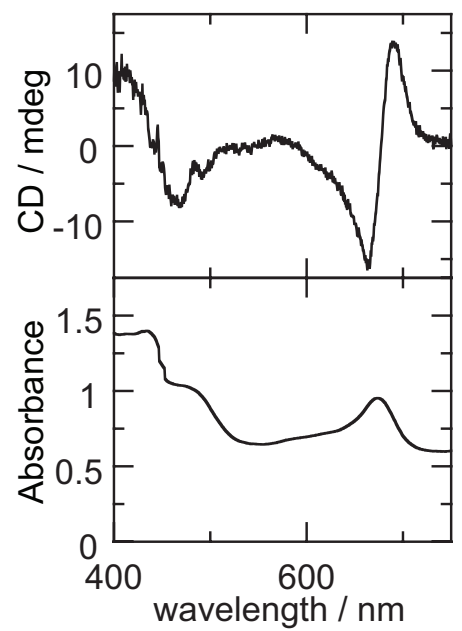

(b)

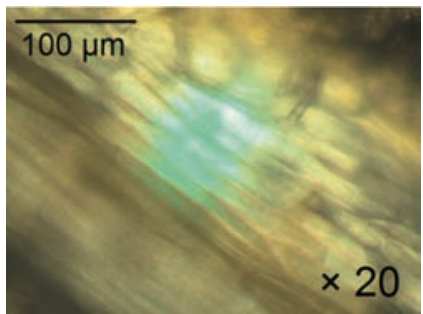

(d)

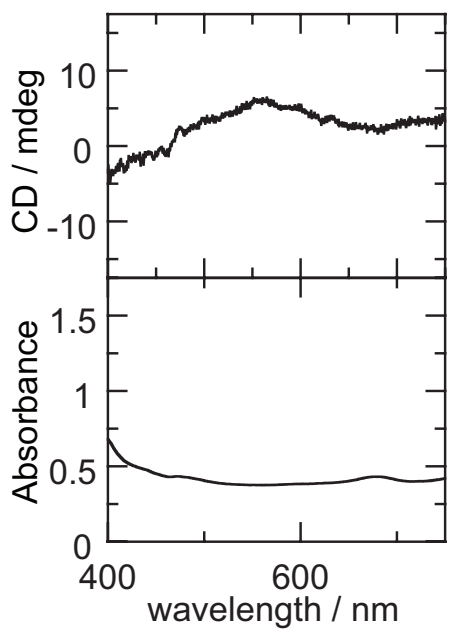

Fig. 4 CCD images, micro-CD and absorption spectra of a leaf of kidney bean, Phaseolus vulgaris. (a) Image of a leaf blade, (b) image of a leaf vein. The green-colored areas in the images are the CD measurement regions, (c) micro-CD and absorption spectra of the leaf blade, (d) micro-CD and absorption spectra of the leaf vein.

\section{Conclusion}

We constructed a microscope device to measure the microscopic circular dichroism spectra, which could be installed in the sample chamber of the conventional CD spectropolarimeter. By using the device, the micro-CD spectra of $60 \times 60 \mu \mathrm{m}$ region of samples could be measured with high quality. The validity of the device was checked by measurements of the CD spectra of both the chiral DNA-TMPyP film on the glass and the leaf tissues of kidney bean. The constructed microscope device will be useful to characterize inhomogeneous samples, such as films with micro-domains and biological samples, which consist of various tissues.

\section{Acknowledgements}

This study was supported by a Grant-in-Aid for Scientific Research (S) (No. 16105002) and in part by "Special Coordination Funds for Promoting Science and Technology: Yuragi Project" of the Ministry of Education, Culture, Sports, Science and Technology, Japan.

\section{References}

1. M. Srinivasarao, Chem. Rev., 1999, 99, 1935.

2. N. Berova, K. Nakanishi, and R. W. Woody (ed.), "Circular Dicroism: Principles and Applications", 2nd ed., 2000, Wiley-VCH, New York.

3. J. H. Yin and H. Watarai, Anal. Sci., 2007, 23, 841.
4. K. Adachi, K. Chayama, and H. Watarai, Chirality, 2006, 18, 599.

5. K. Adachi, K. Chayama, and H. Watarai, Langmuir, 2006, 22,1630 .

6. S. Wada, K. Fujiwara, H. Monjushiro, and H. Watarai, Anal. Sci., 2004, 20, 1489.

7. K. Fujiwara, H. Monjushiro, and H. Watarai, Chem. Phys. Lett., 2004, 394, 349.

8. S. Wada, K. Fujiwara, H. Monjushiro, and H. Watarai, J. Phys.: Condens. Matter, 2007, 19, 375105.

9. M. F. Maestre and J. E. Katz, Biopolymers, 1982, 21, 1899.

10. K. Claborn, E. P. Faucher, M. Kurimoto, W. Kaminsky, and B. Kahr, J. Am. Chem. Sci., 2003, 125, 14825.

11. M. Yamauchi, K. Mawatari, A. Hibara, M. Tokeshi, and T. Kitamori, Anal. Chem., 2006, 78, 2646.

12. R. Hassey, E. J. Swain, N. I. Hammer, D. Venkataraman, and M. D. Barnes, Science, 2006, 314, 1437.

13. R. J. Fiel, J. Biomol. Struct. Dyn., 1989, 6, 1259.

14. L. G. Marzilli, New J. Chem., 1990, 14, 409.

15. R. F. Pasternack and E. J. Gibbs, "Porphyrin and Metalloporphyrin Interactions with Nucleic Acids in Metal Ions in Biological Systems", ed. H. Sigel, 1996, Vol. 33, Marcel Dekker, New York, 367.

16. R. Kuroda and H. Tanaka, J. Chem. Soc. Chem. Commun., 1994, 1575 .

17. U. Sehlstedt, S. K. Kim, P. Carter, J. Goodisman, J. F. Vollano, B. Norden, and J. C. Dabrowiak, Biochemistry, 1994, 33, 417.

18. C. Houssier and K. Sauer, J. Am. Chem. Soc., 1970, 25, 779.

19. T. Watanabe, A. Hongu, K. Honda, M. Nakazato, M. Konno, and S. Saito, Anal. Chem., 1984, 56, 251. 